\title{
A New Finance Chaotic System, its Electronic Circuit Realization, Passivity based Synchronization and an Application to Voice Encryption
}

https://doi.org/10.1515/nleng-2018-0012

Received January 12, 2018; revised April 7, 2018; accepted May 5, 2018.

\begin{abstract}
Modelling and control applications of dynamical systems in chaos theory arising in several areas are investigated and new control techniques are designed in the chaos literature. We propose a new complex finance chaotic model with states as the interest rate, investment demand, and price index. In this work, after studying the dynamical properties of the new finance model, an electronic chaotic circuit of the model is realized in Multisim. Based on passive control theory, we derive a new controller for globally synchronizing state trajectories of the new finance models. Using the new finance chaotic model developed in this work, a new voice encryption algorithm design is presented. With the proposed algorithm, voice encryption application is performed, and results are described.
\end{abstract}

Keywords: Chaos, chaotic systems, circuit design, passive control, voice encryption

PACS: $05.45 . \mathrm{Gg}$

\footnotetext{
*Corresponding Author: Sundarapandian Vaidyanathan, Research and Development Centre, Vel Tech University, Chennai, Tamil Nadu 600062, India, E-mail: sundarcontrol@gmail.com Aceng Sambas, Department of Mechanical Engineering, Universitas Muhammadiyah Tasikmalaya, Indonesia, E-mail: acenx.bts@gmail.com

Sezgin Kacar, Department of Electrical and Electronics Engineering, Faculty of Technology, Sakarya University, Sakarya, Turkey, E-mail: skacar@sakarya.edu.tr

Unal Cavusoglu, Department of Computer Engineering, Faculty of Computer and Information Sciences, Sakarya University, Sakarya, Turkey, E-mail: unalc@sakarya.edu.tr
}

\section{Introduction}

Chaotic dynamical systems and electronic chaotic circuits have applications in many research fields [1-10]. In [1], Vaidyanathan studied a novel chemical chaotic reactor system and its adaptive control. In [2], Vaidyanathan studied the control and synchronization of a 3-D novel jerk chaotic system with two quadratic nonlinearities. In [3], Uthamacumaran investigated a biophysical approach to cancer dynamics with the help of quantum chaos and energy turbulence. In [4], Senouci et al. discussed FPGA implementation of chaotic generators. In [5], Xu et al. studied hysteretic chaotic neuron and its applications in communication systems. In [6], Hu et al. used DNA insertion and DNA deletion for the study of chaotic image cryptosystem. In [7], Yuan et al. studied a 5-D hyperchaotic system and new parallel image cryptosystem. In [8], Zhou et al. studied the cluster synchronization on multiple nonlinearly coupled dynamical subnetworks of complex networks with nonidentical nodes and stochastic perturbations. In [9], Li et al. proposed a hyperchaos based image encryption algorithm using pixel-level permutation and bit-level permutation. In [10], Hamdi et al. proposed a very simple and efficient encryption scheme based on controlled chaotic maps and ADPCM (Adaptive Differential Pulse Code Modulation) coding, to secure the real-time voice communication for operating at 16, 24, 32 or $40 \mathrm{kbps}$.

In 1990, Pecora and Carroll showed that chaotic systems can be synchronized by using a drive-response configuration of chaotic systems in their seminal paper [11]. In recent years, the idea of synchronization of chaotic systems has received a great deal of interest in research areas such as secure communications, encryption, cryptosystems, automatic control, etc. In [12], Sun et al. investigated the synchronization of chaotic PMSM systems for secure communication and parameters identification. In [13], Khorashadizadeh and Majidi proposed a new method for secure communication based on chaos synchronization. In [14], Li et al. proposed a novel image 
encryption algorithm based on synchronized random bit generated in cascade-coupled chaotic semiconductor ring lasers. In [15], Muthukumar et al. studied the sliding mode control design for synchronization of fractional order chaotic systems and its application to a new cryptosystem. In [16], Bhattacharya et al. discussed the implementation of chaotic and synchronization properties of logistic maps using artificial neural networks for code generation. In [17], Cavusoglu et al. described a chaos based Random Number Generator (RNG) which is designed with the help of a new chaotic system and designed a hybrid RSA algorithm for text and image encryption. In [18], Wang et al. described a novel image encryption algorithm that combines the SHA-3 hash function and two chaotic systems, $v i z$. hyperchaotic Lorenz and Chen systems and detailed experimental results of their encryption algorithm. In [19], Jridi and Alfalou deployed chaotic generators to develop a novel chaos-based encryption algorithm. In [20], Nosrati et al. discussed cubature Kalman filter-based chaotic synchronization and image encryption.

Several control techniques such as active control, adaptive control, backstepping control and sliding mode control have been proposed in the literature for the control and synchronization of chaotic systems [21-27].

In recent years, the concept of passivity of nonlinear systems has attracted new interest in nonlinear control theory [28-34]. In engineering, passive control is a scheme of energy dissipation devices and seismic base isolation systems applied to structures to reduce the vibrations without using external power. In [28], Wen used passive control technique for controlling chaos in the Lorenz system. In [29], Song et al. applied passive control technique to design a passive controller to control the NewtonLeipnik chaotic system with multiple strange attractors. In [30], Wang and Liu used passive control technique to study the passive control of a four-scroll chaotic system. In [31], Wang and Liu designed a passive controller to realize the synchronization between two hyperchaotic Lorenz systems under different initial conditions. In [32], Wang and Liu used passive control technique to realize the chaos synchronization problem for the unified chaotic system. In [33], Wu et al. applied the passive control technique to realize the chaos synchronization of Rikitake chaotic attractor. In [34], Kocamaz and Uyaroglu studied the synchronization of Vilnius chaotic oscillators with active and passive control.

Electronic chaotic circuits have important applications in the chaos literature [35-38]. In [35], Pham et al. described a chaotic system having an infinite number of equilibrium points on a rounded square loop and designed an experimental circuit implementing their chaotic model.
In [36], Volos et al. announced their finding of a new fourdimensional hyperchaotic four-wing system and implemented their work with an experimental circuit realization. In [37], Vaidyanathan et al. found a new jerk chaotic circuit and gave an application of their jerk chaotic circuit to voice encryption. In [38], Wang et al. announced a new hyperchaotic hyperjerk system with two polynomial nonlinearities and realized it with an electronic circuit model.

Control and synchronization of finance chaotic systems is an important research area in the chaos literature. In [39], Goa and Ma reported chaos in a new finance chaotic system exhibiting Hopf bifurcation. In [40], Vaidyanathan et al. studied a new finance model describing a complex economical system displaying chaos. In [41], Jian et al. discussed the synchronization of a class of finance chaotic systems. In [42], Cia and Yang studied the globally exponentially attractive set and synchronization of a novel finance chaotic system. In [43], Zhao et al. used Lyapunov stability theory and Routh-Hurwitz stability criteria to design effective controllers for the global asymptotic synchronization of the finance chaotic system. In [44], Li and Xie introduced a modified finance chaotic system and discussed its synchronization via a single controller. In [45], Kocamez et al. carried out a comparative study of the synchronization of chaos in nonlinear finance system by means of sliding mode and passive control methods. In [46], Zheng et. al. investigated a simple hybrid synchronization for a class of finance chaotic systems.

In this work, we propose a new finance chaotic model with state variables as the interest rate, investment demand, and price index. The construction of the new finance chaotic system is carried out by combining two known chaotic models of finance systems [39, 40]. In Section 2, we describe the dynamic equations and properties of the new finance model. In Section 3, we build an electronic circuit of the new finance model with the help of MultiSIM. In Section 4, we derive new results for globally synchronizing the state trajectories of the new finance models via passive control method. In Section 5, a new voice encryption application is performed, and analysis results are given. Section 6 contains the conclusions.

\section{A new finance chaotic model}

Gao and Ma finance model [39] is given by the following dynamical system:

$$
\left\{\begin{array}{l}
\dot{x}_{1}=x_{3}+\left(x_{2}-a\right) x_{1} \\
\dot{x}_{2}=1-b x_{2}-x_{1}^{2} \\
\dot{x}_{3}=-x_{1}-c x_{3}
\end{array}\right.
$$


Vaidyanathan finance model [40] is obtained as a modification of the finance model (1) and described by the following dynamical system:

$$
\left\{\begin{array}{l}
\dot{x}_{1}=x_{3}+\left(x_{2}-a\right) x_{1} \\
\dot{x}_{2}=1-b x_{2}-x_{1}^{4} \\
\dot{x}_{3}=-x_{1}-c x_{3}
\end{array}\right.
$$

In the finance dynamical models (1) and (2), the state variables $x_{1}$ and $x_{2}$ represent the interest rate and investment demand respectively, while the state variable $x_{3}$ represents the price exponent. Also, the financial parameter $a$ represents the household savings and $b$ represents the investment cost, while $c$ stands for the demand elasticity of commercial markets. All the three financial parameters are supposed to be positive.

We note that the Gao-Ma finance model (1) has a quadratic nonlinearity $x_{1} x_{2}$ and another quadratic nonlinearity $x_{1}^{2}$. We also note that the Vaidyanathan finance model (2) has a quadratic nonlinearity $x_{1} x_{2}$ and a quartic nonlinearity $x_{1}^{4}$.

In [39], Gao and Ma showed that the nonlinear finance chaotic system (1) is chaotic when $(a, b, c)=(6,0.1,1)$. The Lyapunov characteristic exponents of the Gao-Ma finance model (1) are obtained in MATLAB for parameter values $(a, b, c)=(6,0.1,1)$, initial state $X(0)=$ $(0.5,3,-0.4)$ and $T=1 E 5$ as $L_{1}=0.090439, L_{2}=0$ and $L_{3}=-0.393061$.

The Kaplan-Yorke dimension of the Gao-Ma finance model (1) is derived as

$$
D_{K Y}=2+\frac{L_{1}+L_{2}}{\left|L_{3}\right|}=2.2301
$$

In [40], Vaidyanathan et al. showed that the nonlinear finance chaotic system (2) is chaotic when $(a, b, c)=$ $(7,0.1,1)$. The Lyapunov characteristic exponents of the Vaidyanathan finance model (2) are obtained in MATLAB for parameter values $(a, b, c)=(7,0.1,1)$, initial state $X(0)=(0.5,3,-0.4)$ and $T=1 E 5$ as $L_{1}=0.125494$, $L_{2}=0$ and $L_{3}=-0.421513$.

The Kaplan-Yorke dimension of the Vaidyanathan finance model (2) is derived as

$$
D_{K Y}=2+\frac{L_{1}+L_{2}}{\left|L_{3}\right|}=2.2977
$$

In this work, we propose a new finance model by combining the Gao-Ma finance model (1) and the Vaidyanathan finance model (2). Specifically, we consider the effect of both quadratic and quartic nonlinearities in the $x_{2}$-dynamics of the system.
The new finance model is described by

$$
\left\{\begin{array}{l}
\dot{x}_{1}=x_{3}+\left(x_{2}-a\right) x_{1} \\
\dot{x}_{2}=1-b x_{2}-d x_{1}^{2}-x_{1}^{4} \\
\dot{x}_{3}=-x_{1}-c x_{3}
\end{array}\right.
$$

In the finance dynamical model (3), the state variables $x_{1}, x_{2}, x_{3}$ and the parameters $a, b, c$ have the same interpretation as in the finance dynamical models (1) and (2). Furthermore, $d$ is a positive constant. The system (5) has two quadratic nonlinearities and a quartic nonlinearity.

The Lyapunov characteristic exponents of the new finance model (5) are obtained in MATLAB for the financial parameter values taken as $(a, b, c, d)=(7.2,0.1,1,0.1)$, initial state values as $X(0)=(0.5,3,-0.4)$ and $T=1 E 5$ as $L_{1}=0.132893, L_{2}=0$ and $L_{3}=-0.412008$.

The Lyapunov characteristic exponents of the new finance system (5) show that the new finance system (5) is chaotic. Since the sum of Lyapunov characteristic exponents is negative, the new finance chaotic system (5) is dissipative with a strange chaotic oscillator.

The Kaplan-Yorke dimension of the new finance chaotic system (5) is determined as follows:

$$
D_{K Y}=2+\frac{L_{1}+L_{2}}{\left|L_{3}\right|}=2.3225
$$

Table 1 shows a comparison of the Gao-Ma finance system (1), Vaidyanathan finance system (2) and the new finance system (5). From Table 1, we see that the new finance chaotic system (5) is more chaotic than the Gao-Ma finance system (1) and Vaidyanathan finance system (2).

Next, we note that the new finance chaotic system (5) stays invariant under the coordinates transformation

$$
\left(x_{1}, x_{2}, x_{3}\right) \mapsto\left(-x_{1}, x_{2},-x_{3}\right)
$$

for all values of the system parameters $(a, b, c, d)$. This shows that the new finance chaotic system (5) has rotation symmetry about the $x_{2}$-axis. Hence, for every non-trivial trajectory $\left(x_{1}(t), x_{2}(t), x_{3}(t)\right)$ of the system (5), $\left(-x_{1}(t), x_{2}(t),-x_{3}(t)\right)$ is also a trajectory of the system (5).

Figures 1-3 illustrate the two-dimensional phase plots of the new finance chaotic system (5). Figure 4 displays the Lyapnov characteristic exponents of the new finance chaotic system (5) for the parameter $d$. For these numerical simulations, we have taken the initial state as $X(0)=$ $(0.5,3,-0.4)$ and the parameter values as $(a, b, c, d)=$ $(7.2,0.1,1,0.1)$ for the new finance chaotic system (5).

The equilibrium points of the new finance chaotic system (5) are obtained by solving the following system of equations.

$$
x_{3}+\left(x_{2}-a\right) x_{1}=0
$$


Table 1: A Comparison of the Finance Chaotic Systems

\begin{tabular}{clll}
\hline Chaotic System & \multicolumn{1}{c}{ Lyapunov Exponents } & \multicolumn{1}{c}{$\begin{array}{c}\text { Maximal Lyapunov } \\
\text { Exponent (MLE) }\end{array}$} & $\begin{array}{l}\text { Kaplan-Yorke } \\
\text { Dimension }\end{array}$ \\
\hline Gao-Ma Finance System [39] & $\begin{array}{l}L_{1}=0.090439, L_{2}=0, \\
L_{3}=-0.393061\end{array}$ & $L_{1}=0.090439$ & $D_{K Y}=2.2301$ \\
\hline $\begin{array}{c}\text { Vaidyanathan Finance } \\
\text { System [40] }\end{array}$ & $\begin{array}{l}L_{1}=0.125494, L_{2}=0, \\
L_{3}=-0.421513\end{array}$ & $L_{1}=0.125494$ & $D_{K Y}=2.2977$ \\
\hline New Finance System & $\begin{array}{l}L_{1}=0.132893, L_{2}=0, \\
L_{3}=-0.412008\end{array}$ & $L_{1}=0.132893$ & $D_{K Y}=2.3225$ \\
\hline
\end{tabular}

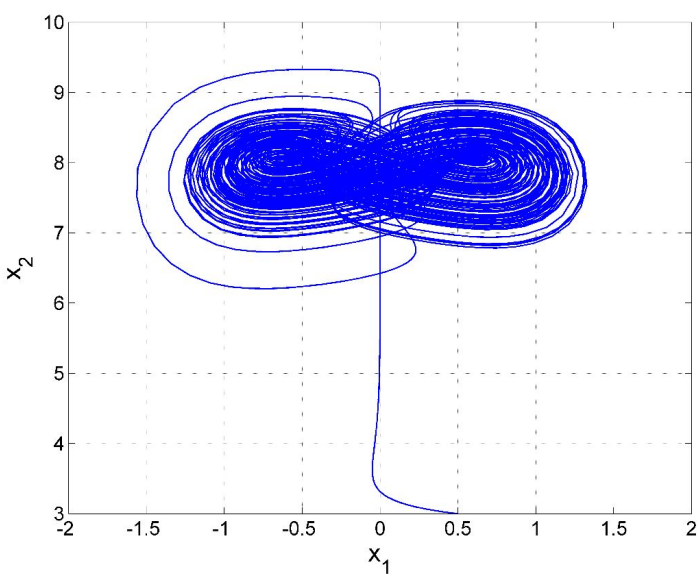

Fig. 1: Numerical simulations of the 2-D phase plot in $\left(x_{1}, x_{2}\right)$-plane of the new finance chaotic system (5) for $X(0)=(0.5,3,-0.4)$ and $(a, b, c, d)=(7.2,0.1,1,0.1)$

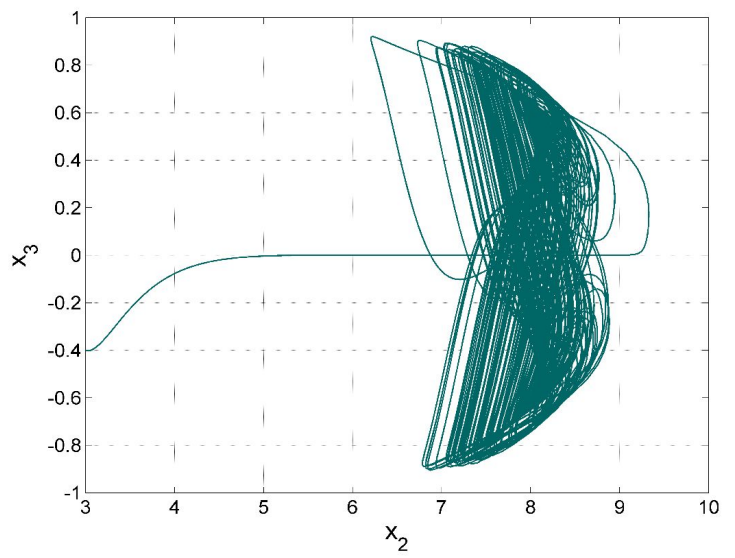

Fig. 2: Numerical simulations of the 2-D phase plot in $\left(x_{2}, x_{3}\right)-$ plane of the new finance model (3) for $X(0)=(0.5,3,-0.4)$ and $(a, b, c, d)=(7.2,0.1,1,0.1)$

$$
\begin{gathered}
1-b x_{2}-d x_{1}^{2}-x_{1}^{4}=0 \\
-x_{1}-c x_{3}=0
\end{gathered}
$$

From Eq. (8c), it follows that

$$
x_{1}=-c x_{3}
$$

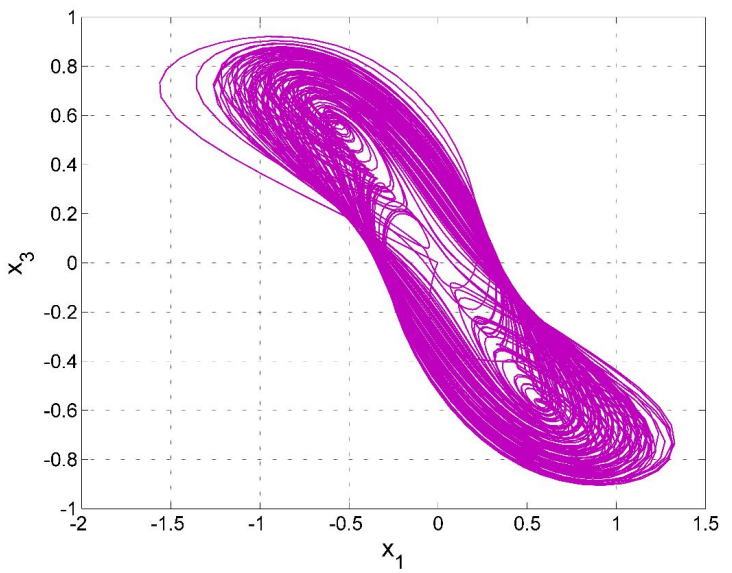

Fig. 3: Numerical simulations of the 2-D phase plot in $\left(x_{1}, x_{3}\right)$-plane of the new finance model (3) for $X(0)=(0.5,3,-0.4)$ and $(a, b, c, d)=(7.2,0.1,1,0.1)$

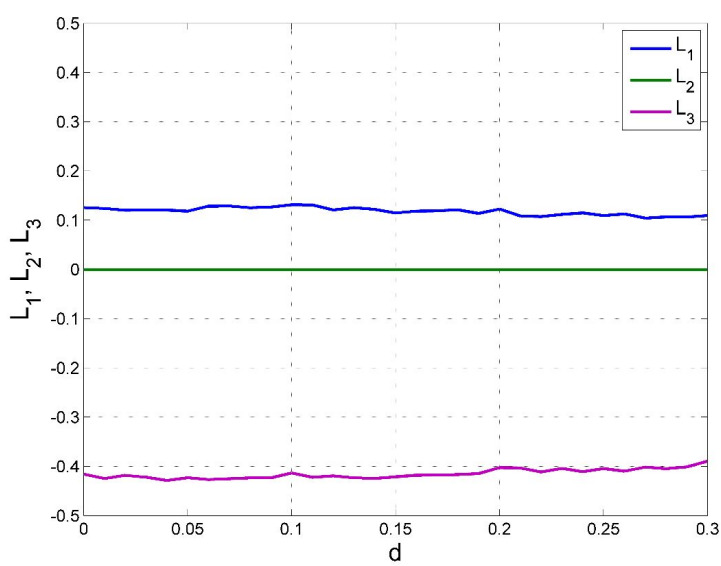

Fig. 4: Lyapunov characteristic exponents for the parameter $d$ of the new finance chaotic system (5), where $X(0)=(0.5,3,-0.4)$ and $(a, b, c)=(7.2,0.1,1)$

From (8a) and (9), we get

$$
x_{3}-c x_{3}\left(x_{2}-a\right)=0
$$

We have two cases to consider: (i) $x_{3}=0$ and (ii) $x_{3} \neq 0$.

In Case (i), we let $x_{3}=0$. From (9), $x_{1}=0$. Substituting $x_{1}=x_{3}=0$ in Eq. (8b), we get $1-b x_{2}=0$. Thus, it is easy 
to see that the new chaotic system (5) has the equilibrium

$$
E_{0}=\left[\begin{array}{c}
0 \\
1 / b \\
0
\end{array}\right]
$$

In Case (ii), we let $x_{3} \neq 0$. From Eq. (10), we must have $1-c\left(x_{2}-a\right)=0$. Thus, we have

$$
x_{2}=a+\frac{1}{c}=\mu \text { (say) }
$$

Let $z=x_{1}^{2}$. Then we can write Eq. (8b) as the quadratic equation

$$
z^{2}+d z+(b \mu-1)=0
$$

Solving (13), we get

$$
z=\frac{-d \pm \sqrt{d^{2}-4(b \mu-1)}}{2}
$$

In order that $z>0$, we must have $b \mu-1<0$ or $\mu<\frac{1}{b}$. In this case, we get

$$
z=x_{1}^{2}=\frac{-d+\sqrt{d^{2}-4(b \mu-1)}}{2}
$$

Thus, when $\mu=a+\frac{1}{c}<\frac{1}{b}$, the new chaotic system (5) has two more equilibrium points

$$
E_{1}=\left[\begin{array}{c}
\frac{1}{\sqrt{2}}\left[-d+\sqrt{d^{2}-4(b \mu-1)}\right]^{0.5} \\
\mu \\
\frac{-1}{c \sqrt{2}}\left[-d+\sqrt{d^{2}-4(b \mu-1)}\right]^{0.5}
\end{array}\right]
$$

and

$$
E_{2}=\left[\begin{array}{c}
\frac{-1}{\sqrt{2}}\left[-d+\sqrt{d^{2}-4(b \mu-1)}\right]^{0.5} \\
\mu \\
\frac{1}{c \sqrt{2}}\left[-d+\sqrt{d^{2}-4(b \mu-1)}\right]^{0.5}
\end{array}\right]
$$

Combining the cases (i) and (ii), we obtain three equilibrium points of the new finance chaotic system $E_{0}, E_{1}$ and $E_{2}$, which are defined in the equations (11) and (16).

For the parameter values $(a, b, c, d)$ $(7.2,0.1,1,0.1)$, the new finance chaotic system (5) has three equilibrium points, which are derived as follows:

$$
\begin{aligned}
& E_{0}=\left[\begin{array}{c}
0 \\
10 \\
0
\end{array}\right], E_{1}=\left[\begin{array}{c}
0.6142 \\
8.2 \\
-0.6142
\end{array}\right] \\
& \text { and } E_{2}=\left[\begin{array}{c}
-0.6142 \\
8.2 \\
0.6142
\end{array}\right]
\end{aligned}
$$

We can express the new finance chaotic system (5) in vector notation as follows:

$$
\dot{X}=f(X) \text {, where } f(X)=\left[\begin{array}{c}
x_{3}+\left(x_{2}-a\right) x_{1} \\
1-b x_{2}-d x_{1}^{2}-x_{1}^{4} \\
-x_{1}-c x_{3}
\end{array}\right]
$$

We find that

$$
J_{0}=D f\left(E_{0}\right)=\left[\begin{array}{ccc}
2.8 & 0 & 1 \\
0 & -0.1 & 0 \\
-1 & 0 & -1
\end{array}\right]
$$

which has the spectral values

$$
\lambda_{1}=-0.1, \lambda_{2}=-0.7155, \lambda_{3}=2.5155
$$

Thus, the equilibrium $E_{0}$ is a saddle point and unstable.

A simple calculation shows that

$$
J_{1}=D f\left(E_{1}\right)=\left[\begin{array}{ccc}
1.0000 & 0.6142 & 1 \\
-1.0496 & -0.1 & 0 \\
-1 & 0 & -1
\end{array}\right]
$$

which has the spectral values

$$
\lambda_{1}=0.2133, \lambda_{2,3}=0.8434 \pm 1.1720 i
$$

Thus, the equilibrium $E_{1}$ is a saddle-focus and unstable. A similar calculation also shows that $E_{2}$ is a saddlefocus and unstable.

Figures 5, 6 and 7 describe the calculation of Lyapunov characteristic exponents and the bifurcation diagrams for the new system (5) with respect to the three system parameters $a, b$ and $c$, respectively.

Our bifurcation analysis is described as follows.

\section{Case (A): Effect of varying $a$}

Here, we fix $b=0.1, c=1, d=0.1$ and vary $a$. When $7.20 \leq a \leq 8.20$, the new system (5) displays chaotic behavior. Near $a=8.21$, the system (5) displays periodic behavior. For the region $8.22 \leq a \leq 8.30$, the system (5) displays chaotic behavior. Also, when $a>8.30$, the system (5) displays periodic behavior. The bifurcation analysis for the case of varying $a$ is described by Figure 5 .

\section{Case (B): Effect of varying $b$}

Here, we fix $a=7.2, c=1, d=0.1$ and vary $b$. When $0 \leq b \leq 0.067$, the new system (5) exhibits periodic behaviour. When $0.068 \leq b \leq 0.113$, the system (5) displays chaotic behaviour. Also, when $b \geq 0.114$, the system (5) displays a fixed point. The bifurcation analysis for the case of varying $b$ is described by Figure 6 .

\section{Case (C): Effect of varying $c$}

Here, we fix $a=7.2, b=0.1, d=0.1$ and vary $c$. When $0 \leq c \leq 0.90$, the new system (5) displays periodic behavior. When $0.91 \leq c \leq 1.56$, the system (5) displays chaotic behaviour. Also, when $c \geq 1.57$, the system (5) displays periodic behaviour. The bifurcation analysis for the case of varying $c$ is described by Figure 7 . 

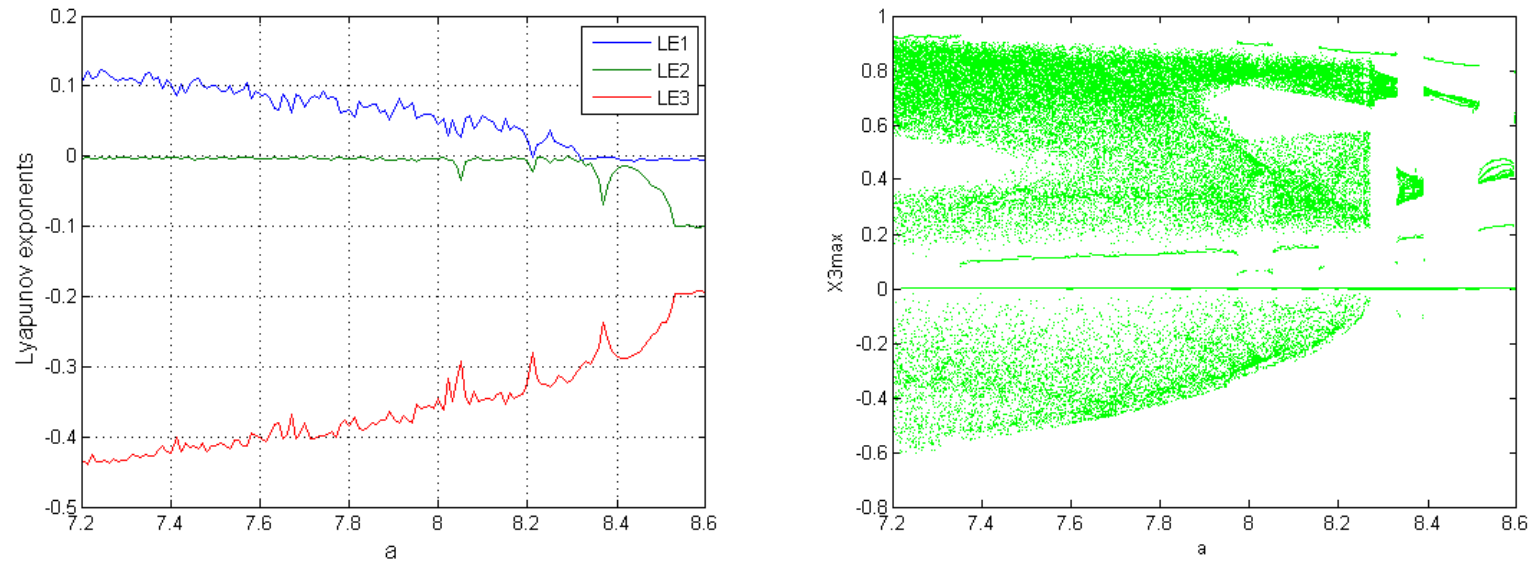

Fig. 5: Diagram of the Lyapunov characteristic exponents versus $a$ and the bifurcation diagram of X3max versus $a$ for $b=0.1, c=1$ and $d=0.1$
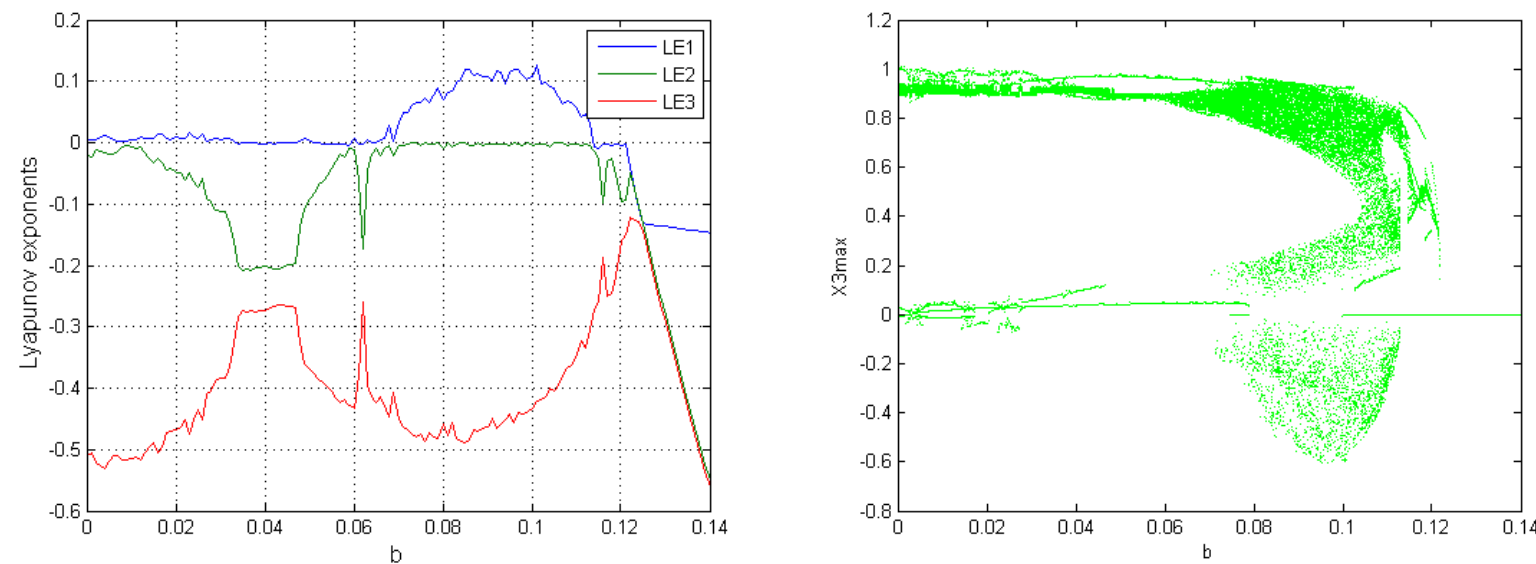

Fig. 6: Diagram of the Lyapunov characteristic exponents versus $b$ and the bifurcation diagram of X3max versus $b$ for $a=7.2, c=1$ and $d=0.1$
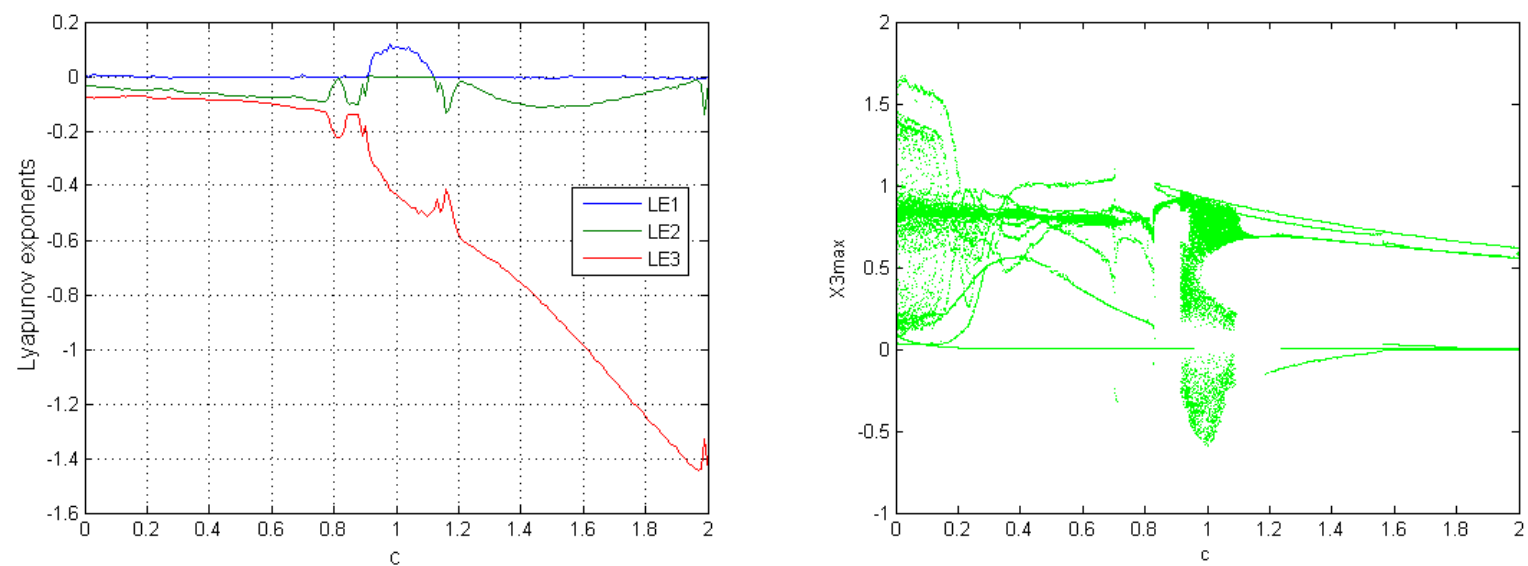

Fig. 7: Diagram of the Lyapunov characteristic exponents versus $c$ and the bifurcation diagram of X3max versus $c$ for $a=7.2, b=0.1$ and $d=0.1$ 


\section{Circuit realization of the new finance chaotic model}

In this section, the electronic circuit design of the new finance model (5) is constructed. In this study, a linear scaling is considered as follows:

$$
\left\{\begin{array}{l}
\dot{x}_{1}=x_{3}+\left(4 x_{2}-a\right) x_{1} \\
\dot{x}_{2}=\frac{1}{4}-b x_{2}-4 d x_{1}^{2}-64 x_{1}^{4} \\
\dot{x}_{3}=-x_{1}-c x_{3}
\end{array}\right.
$$

By applying Kirchhoff's laws to this circuit, we get the following circuital equations:

$$
\left\{\begin{array}{l}
\frac{d V c_{1}}{d t}=\frac{1}{C_{1} R_{1}} V c_{3}+\frac{1}{C_{1} R_{2}} V c_{1} V c_{2}-\frac{1}{C_{1} R_{3}} V c_{1} \\
\frac{d V c_{2}}{d t}=\frac{1}{C_{2} R_{4}} V 1-\frac{1}{C_{2} R_{5}} V c_{2}-\frac{1}{C_{2} R_{6}} V c_{1}{ }^{2}-\frac{1}{C_{2} R_{7}} V c_{1}{ }^{4} \\
\frac{d V c_{3}}{d t}=-\frac{1}{C_{3} R_{8}} V c_{1}-\frac{1}{C_{3} R_{9}} V c_{3}
\end{array}\right.
$$

In Eq. (7), $C_{1}, C_{2}, C_{3}$ are the capacitors and $V_{C_{1}}, V_{C_{2}}, V_{C_{3}}$ are the voltages across them. Also, each state of the finance model (5), i.e. $x_{1}, x_{2}, x_{3}$ is implemented as the voltage across the corresponding capacitors $C_{1}, C_{2}$ and $C_{3}$, respectively.

We choose the values of the circuital elements as: $R_{1}=$ $R_{8}=R_{9}=400 \mathrm{k} \Omega, R_{3}=55.55 \mathrm{k} \Omega, R_{6}=1 \mathrm{M} \Omega, R_{5}=4 \mathrm{M} \Omega, R_{7}=$ $6.25 \mathrm{k} \Omega, \mathrm{R}_{4}=1.6 \mathrm{k} \Omega, \mathrm{V}_{1}=-1 \mathrm{~V}_{D C}, \mathrm{R}_{2}=\mathrm{R}_{10}=\mathrm{R}_{11}=\mathrm{R}_{12}=\mathrm{R}_{13}=$ $100 \mathrm{k} \Omega, \mathrm{C}_{1}=\mathrm{C}_{2}=\mathrm{C}_{3}=1 \mathrm{nF}$.

The supplies of all active devices are \pm 15 Volt. Using the circuit design approach based on the operational amplifiers [47-53], we have designed the electronic chaotic circuit as shown in Figure 8. The circuit simulations of the phase plots are displayed in Figures 9-11. Clearly, the Multisim output results in Figures 9-11 show a good match with the MATLAB simulations in Figures 1-3.

\section{Passive control design for the complete synchronization of the new finance chaotic model}

This section describes our new results for the complete synchronization of a pair of identical new finance chaotic models (taken as the master and slave systems) via passive control.

As the master system, we take the new finance model with the following dynamics:

$$
\left\{\begin{array}{l}
\dot{\xi}_{1}=\xi_{3}+\left(\xi_{2}-a\right) \xi_{1} \\
\dot{\xi}_{2}=1-b \xi_{2}-d \xi_{1}^{2}-\xi_{1}^{4} \\
\dot{\xi}_{3}=-\xi_{1}-c \xi_{3}
\end{array}\right.
$$

As the slave system, we take the new controlled finance model on with the following dynamics:

$$
\left\{\begin{array}{l}
\dot{\eta}_{1}=\eta_{3}+\left(\eta_{2}-a\right) \eta_{1}+u \\
\dot{\eta}_{2}=1-b \eta_{2}-d \eta_{1}^{2}-\eta_{1}^{4} \\
\dot{\eta}_{3}=-\eta_{1}-c \eta_{3}
\end{array}\right.
$$

The synchronization between the finance models (25) and (26) is quantified by the synchronization error defined by the following equations:

$$
\left\{\begin{array}{l}
e_{1}=\eta_{1}-\xi_{1} \\
e_{2}=\eta_{2}-\xi_{2} \\
e_{3}=\eta_{3}-\xi_{3}
\end{array}\right.
$$

The time-evolution of the error trajectories is governed by the following differential equation:

$$
\left\{\begin{array}{l}
\dot{e}_{1}=e_{3}-a e_{1}+\eta_{1} \eta_{2}-\xi_{1} \xi_{2}+u \\
\dot{e}_{2}=-b e_{2}-e_{1}\left(\xi_{1}+\eta_{1}\right)\left(d+\xi_{1}^{2}+\eta_{1}^{2}\right) \\
\dot{e}_{3}=-e_{1}-c e_{3}
\end{array}\right.
$$

Suppose that $y=e_{1}$ is the output of the error dynamics system (28).

We also define new states as $z_{1}=e_{2}$ and $z_{2}=e_{3}$. Then it is possible to express the error dynamical system (28) in normal form as follows.

$$
\left\{\begin{array}{l}
\dot{z}_{1}=-b z_{1}-\left(\xi_{1}+\eta_{1}\right)\left(d+\xi_{1}^{2}+\eta_{1}^{2}\right) y \\
\dot{z}_{2}=-y-c z_{2} \\
\dot{y}=z_{2}-a y+\eta_{1} \eta_{2}-\xi_{1} \xi_{2}+u
\end{array}\right.
$$

We express the system (29) in the standard form of passive control theory [54] as follows:

$$
\left\{\begin{array}{l}
\dot{Z}=f_{0}(Z)+p(Z, y) y \\
\dot{y}=\beta(Z, y)+\alpha(Z, y) u
\end{array}\right.
$$

where

$$
\left\{\begin{array}{l}
f_{0}(Z)=\left[\begin{array}{l}
-b z_{1} \\
-c z_{2}
\end{array}\right], p(Z, y)=\left[\begin{array}{c}
-\left(\xi_{1}+\eta_{1}\right)\left(d+\xi_{1}^{2}+\eta_{1}^{2}\right) \\
-1
\end{array}\right] \\
\beta(Z, y)=z_{2}-a y+\eta_{1} \eta_{2}-\xi_{1} \xi_{2}, \alpha(Z, y)=1
\end{array}\right.
$$

We consider

$$
W(Z)=\frac{1}{2}\left(z_{1}^{2}+z_{2}^{2}\right)
$$

We find the time-derivative of $W(Z)$ along the dynamics

$$
\dot{Z}=f_{0}(Z)
$$

We find that

$$
\dot{W}=\frac{\partial W}{\partial Z} f_{0}(Z)=-b z_{1}^{2}-c z_{2}^{2}
$$




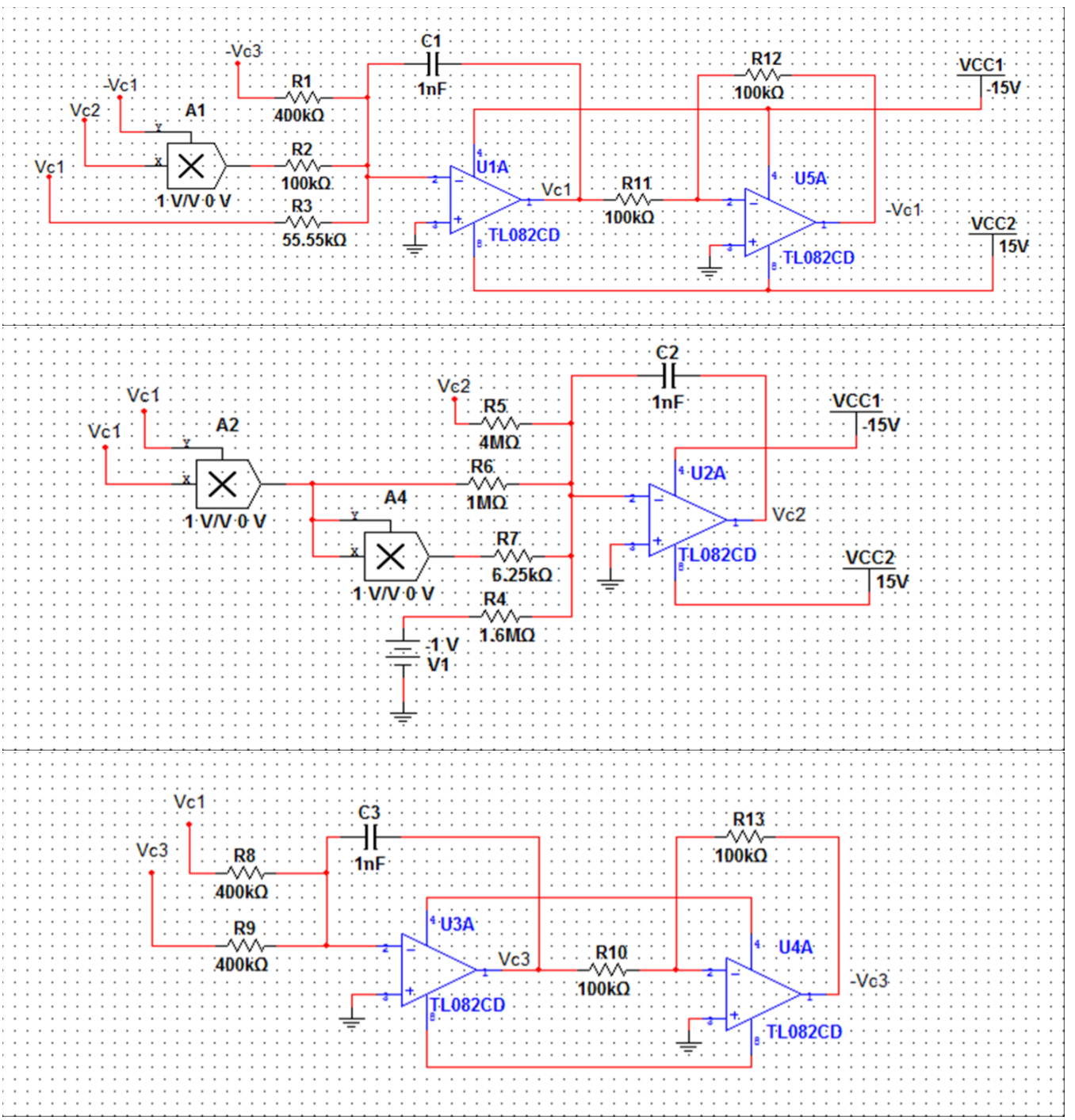

Fig. 8: Circuit realization of the new finance model (5)

We choose the storage function by the relation

$$
V(Z, y)=W(Z)+\frac{1}{2} y^{2}
$$

Clearly, $V$ is positive definite on $R^{3}$.

The time-derivative of $V$ along the error dynamics (14) is found as follows:

$$
\dot{V}=-b z_{1}^{2}-c z_{2}^{2}+y[A+u]
$$

where

$$
A=-\left(\xi_{1}+\eta_{1}\right)\left(d+\xi_{1}^{2}+\eta_{1}^{2}\right) z_{1}-a y+\eta_{1} \eta_{2}-\xi_{1} \xi_{2}
$$

As the passive controller $u$, we choose

$$
u=\left(\xi_{1}+\eta_{1}\right)\left(d+\xi_{1}^{2}+\eta_{1}^{2}\right) z_{1}+(a-k) y-\eta_{1} \eta_{2}+\xi_{1} \xi_{2}
$$

where $k>0$ is a positive gain constant.
Putting (38) into (36), we find that

$$
\dot{V}=-b z_{1}^{2}-c z_{2}^{2}-k y^{2},
$$

which is a negative definite function on $R^{3}$.

By Lyapunov stability theory [55], it is deduced that the synchronization error dynamics (29) is globally asymptotically stable for all initial conditions.

Hence, we have proved the following control result.

Theorem 1. The passive control (38) with $k>0$ 0achieves global and complete asymptotic synchronization of the new finance chaotic models (25) and (26) for all $\xi(0), \eta(0) \in R^{3}$.

For MATLAB simulations, we take the financial parameters as in the chaotic case, viz. $(a, b, c, d)=$ $(7.2,0.1,1,0.1)$. Also, as gain constant, we choose $k=$ 0.5 . 


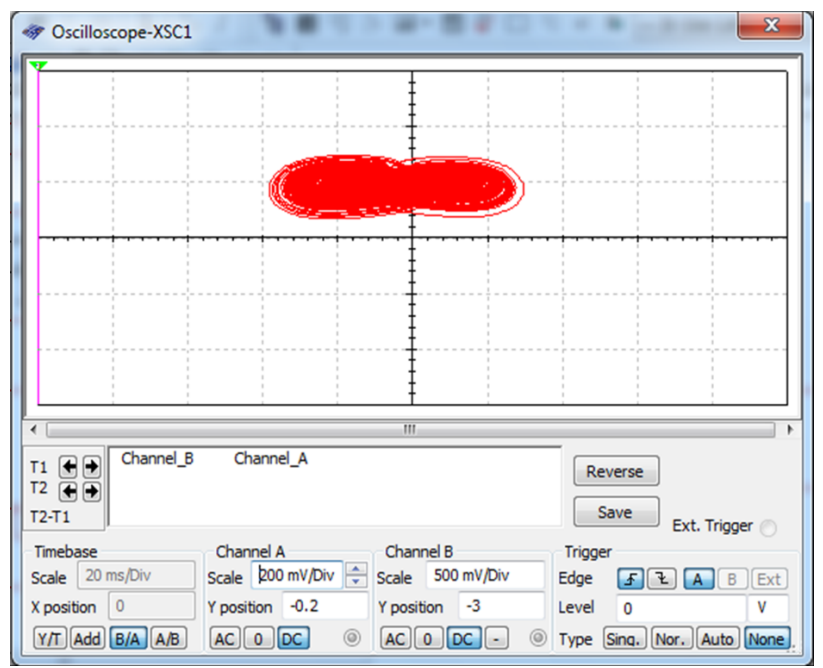

Fig. 9: MultiSIM plot of the new finance model (5) in $x_{1}-x_{2}$ plane

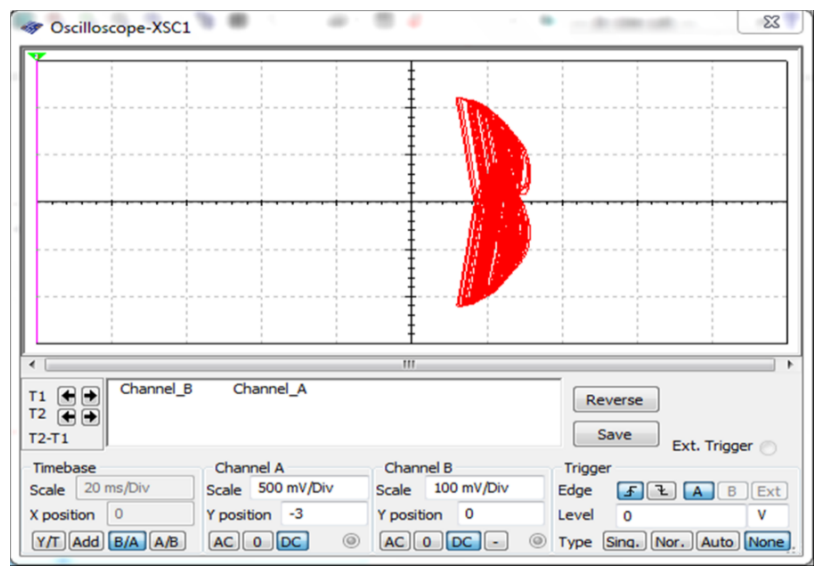

Fig. 10: MultiSIM plot of the new finance model (5) in $x_{2}-x_{3}$ plane

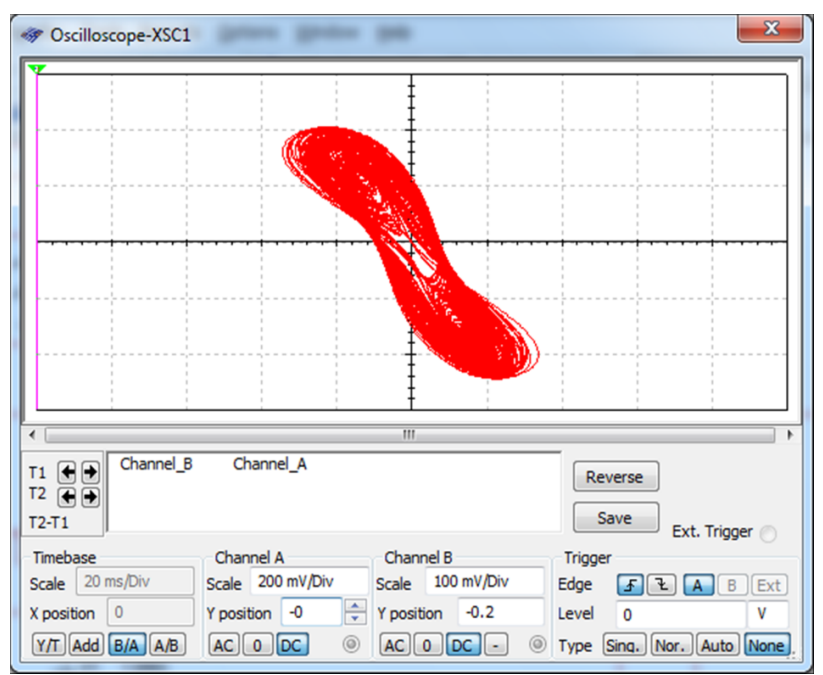

Fig. 11: MultiSIM plot of the new finance chaotic system (5) in $x_{1}-x_{3}$ plane
The initial state of the master system (25) is taken as $\xi(0)=(1.2,0.8,1.7)$ and that of the slave system (26) is taken as $\eta(0)=(0.6,1.4,0.9)$. Figure 12 displays the asymptotic synchronization of the new finance models (25) and (26). Figure 13 displays the time-history of the synchronization errors between the finance models (25) and (26).
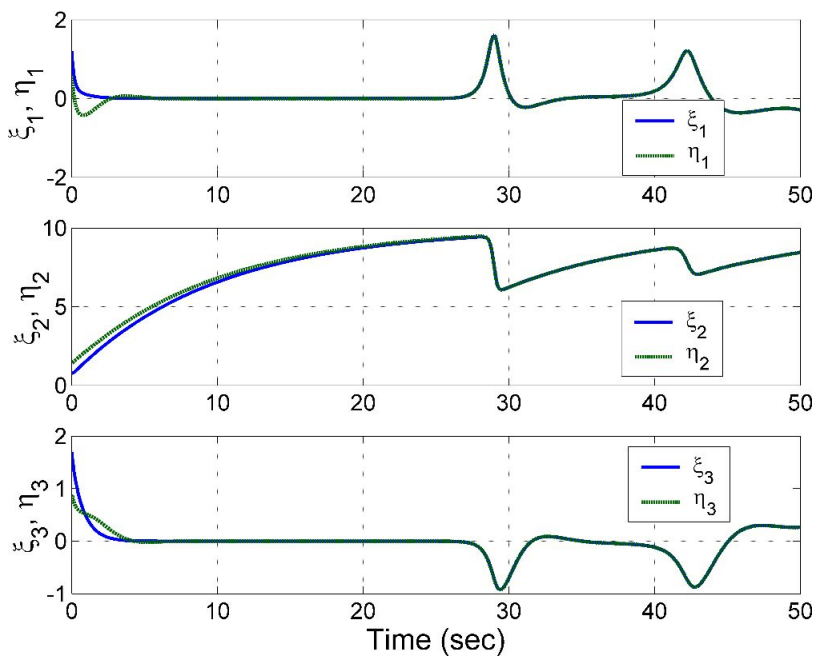

Fig. 12: MATLAB simulation demonstrating the asymptotic synchronization of the new finance models (9) and (10) for $\xi(0)=$ $(1.2,0.8,1.7)$ and $\eta(0)=(0.6,1.4,0.9)$

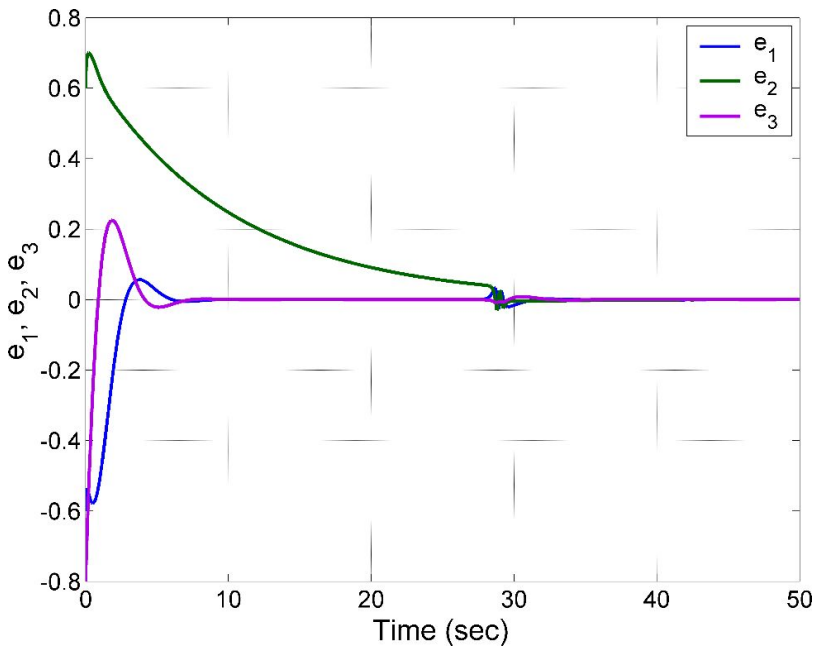

Fig. 13: MATLAB simulation depicting the time-history of the synchronization errors between the new finance models (9) and (10) for $\xi(0)=(1.2,0.8,1.7)$ and $\eta(0)=(0.6,1.4,0.9)$ 


\section{Voice encryption application and its analysis}

\subsection{Voice encryption algorithm design}

Using the new finance model (5) developed in this work, voice encryption is performed. The generation of random numbers to be used in the voice encryption process has been performed. After entering the initial conditions of the chaotic model and system parameters in the random number generation, the chaotic model is solved by the classical fourth-order Runge-Kutta (RK-4) method and the float number values are obtained. With the conversion of the float numbers obtained from all three phases to the binary system, random bit sequences are generated. The length of the generated random bit array depends on the length of the voice file. For voice file encryption, it is converted to binary form from binary. In a binary format voice file is subjected to XOR processing with the bit sequence obtained from the random number generator. After the encryption process, the data is converted to a float form and an encrypted voice file is obtained. On the receiving side, the same random number is obtained with the identical random number generator, and the original voice file is obtained by decrypting the encrypted voice file. Figure 14 depicts the block diagram of the voice encryption algorithm.

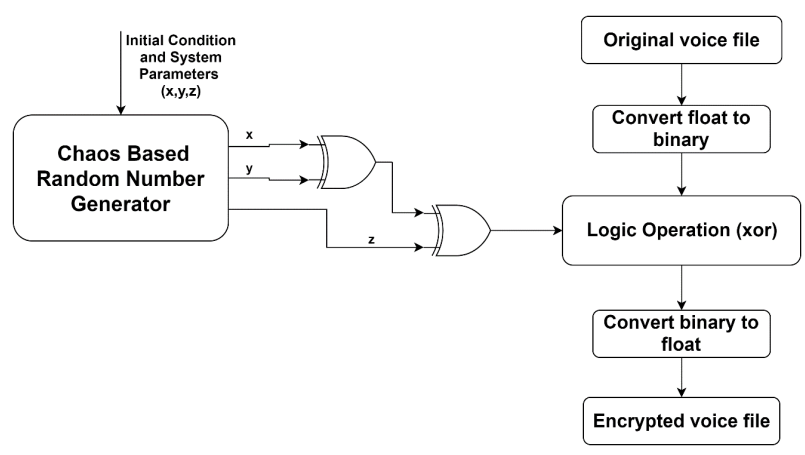

Fig. 14: The voice encryption block diagram

\subsection{Voice encryption application and its analysis}

After the design of the encryption algorithm, voice encryption application is implemented. Figure 15 shows the original, encrypted and decrypted voice files used in the en-

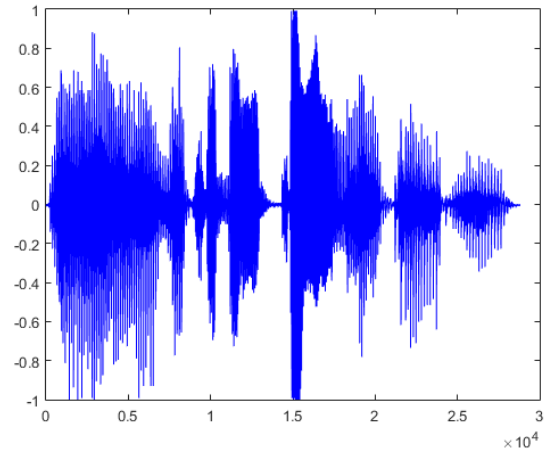

(a)

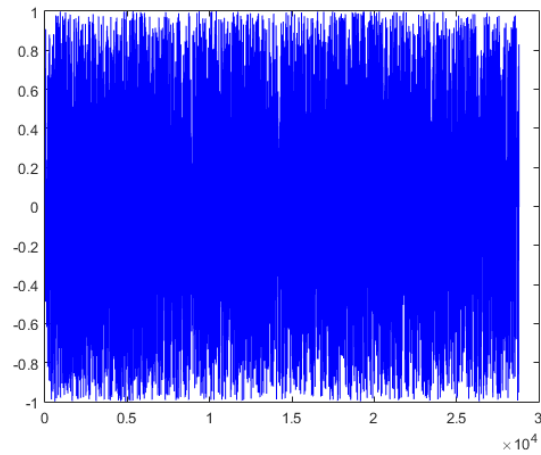

(b)

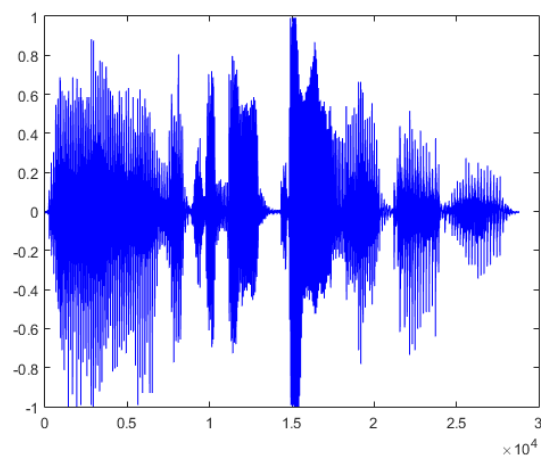

(c)

Fig. 15: Original, encrypted and decrypted voice files

cryption process. When we analyse at the graph of the encrypted voice file, it appears that a completely different voice file is obtained from the original file in Figure 15(a). When Figure 15(a) and (c) are examined, it is seen that the voice file has been successfully decrypted and the original voice file has been obtained. Figure 16 shows frequency spectrum analysis results of original, encrypted and decrypted voice files. When Figure 16(a) and (b) are examined, it is seen that the spectrum graph of the encrypted voice file has much wider frequency values than the original voice file of spectrum analysis results and has almost 
equal spectrum values for each sampling value. In Figures 16(a) and (c), it is seen that the spectrum analyses of the original and the decrypted voice file are equal. According to the results of frequency spectrum analysis, it has been found that the proposed encryption algorithm successfully performs voice encryption and decryption.

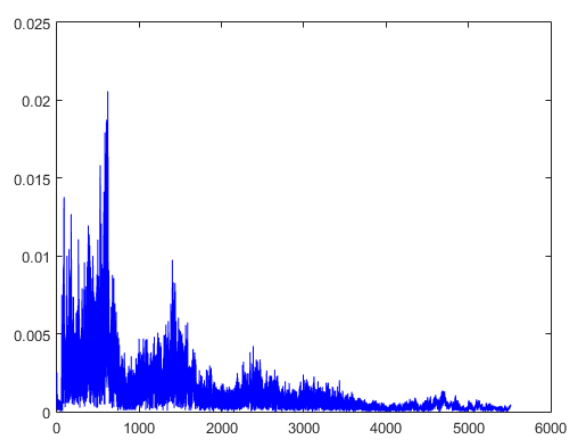

(a)

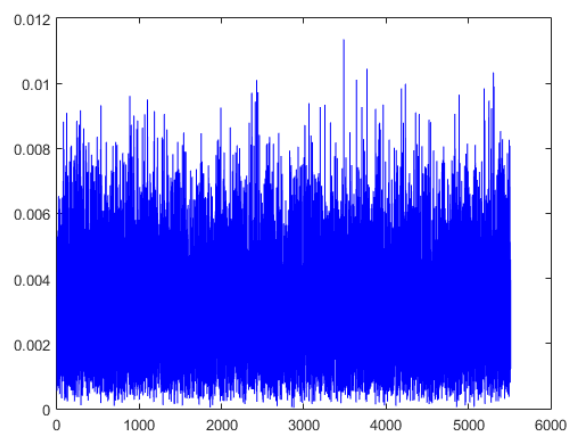

(b)

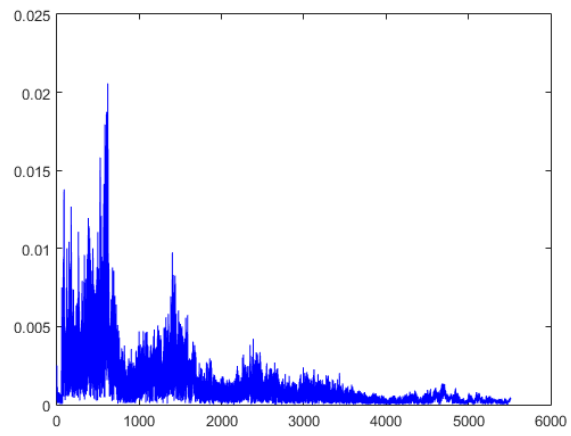

(c)

Fig. 16: The frequency spectrum analysis results of the original, encrypted and decrypted voice files

\section{Conclusions}

This work reported the finding a new complex finance chaotic model with state variables consisting of the interest rate, investment demand, and price index. The dynamical properties of the new finance chaotic model were studied in detail. An electronic chaotic circuit of the new finance model was designed and analyzed in Multisim. Using passive control theory, we developed a new controller for the global and asymptotic synchronization of new finance chaotic models. Numerical simulations were displayed to elucidate all the main results presented in this work. In this study, a new voice encryption algorithm is presented using random numbers generated by a random number generator designed with the new finance chaotic model. Voice encryption application is performed the new algorithm and frequency spectrum analysis of encryption process is performed. According to the analysis results, it has been determined that the proposed encryption algorithm successfully performs the encryption process.

\section{References}

[1] S. Vaidyanathan, "A novel chemical chaotic reactor system and its adaptive control," International Journal of ChemTech Research, vol. 8, no. 7, pp. 146-158, 2015.

[2] S. Vaidyanathan, "Analysis, control, and synchronization of a 3-D novel jerk chaotic system with two quadratic nonlinearities," Kyungpook Mathematical Journal, vol. 55, no. 3, pp. 563-586, 2015.

[3] A. Uthamacumaran, "A biophysical approach to cancer dynamics: Quantum chaos and energy turbulence," Biosystems, vol. 156-157, pp.1-22, 2017.

[4] A. Senouci, H. Bouhedjeur, K. Tourche and A. Boukabou, "FPGA based hardware and device-independent implementation of chaotic generators," AEU-International Journal of Electronics and Communications, vol. 82, pp. 211-220, 2017.

[5] G. Xu, J. Xu, C. Xiu, F. Liu and Y. Zang, "Secure communication based on the synchronous control of hysteretic chaotic neuron," Neurocomputing, vol. 227, pp. 108-112, 2017.

[6] T. Hu, Y. Liu, L.-H. Gong, S.-F. Guo and H.-M. Yuan, "Chaotic image cryptosystem using DNA deletion and DNA insertion," Signal Processing, vol. 134, pp. 234-243, May 2017.

[7] H.-M. Yuan, Y. Liu, T. Lin, T. Hu and L.-H. Gong, "A new parallel image cryptosystem based on 5D hyper-chaotic system," Signal Processing: Image Communication, vol. 52, pp. 87-96, March 2017.

[8] L. Zhou, C. Wang, S. Du and L. Zhou, “Cluster synchronization on multiple nonlinearly coupled dynamical subnetworks of complex networks with nonidentical nodes," IEEE Transactions on Neural Networks and Learning Systems, vol. 28, no. 3, pp. 570-583, 2017. 
[9] Y. Li, C. Wang and H. Chen, "A hyper-chaos-based image encryption algorithm using pixel-level permutation and bit-level permutation," Optics and Lasers in Engineering, vol. 90, pp. 238-246, 2017.

[10] M. Hamdi, R. Rhouma and S. Belghith, “An appropriate system for securing real-time voice communication based on ADPCM coding and chaotic maps," Multimedia Tools and Applications, vol. 76, no. 5, pp. 7105-7128, 2017.

[11] L.M. Pecora and T.L. Carroll, "Synchronization in chaotic systems," Physical Review Letters, vol. 64, no. 8, pp. 821-824, 1990.

[12] Z. Sun, L. Si, Z. Shang and J. Lei, "Finite-time synchronization of chaotic PMSM systems for secure communication and parameters identification,” Optik, vol. 157, pp. 43-55, 2018.

[13] S. Khorashadizadeh and M.H. Majidi, "Chaos synchronization using the Fourier series expansion with application to secure communications," AEU-International Journal of Electronics and Communications, vol. 82, pp. 37-44, 2017.

[14] J. Li, S. Xiang, H. Wang, J. Gong and A. Wen, “A novel image encryption algorithm based on synchronized random bit generated in cascade-coupled chaotic semiconductor ring lasers," Optics and Lasers in Engineering, vol. 102, pp. 1339-1351, 2018.

[15] P. Muthukumar, P. Balasubramaniam and K. Ratnavelu, "Sliding mode control design for synchronization of fractional order chaotic systems and its application to a new cryptosystem," International Journal of Dynamics and Control, vol. 5, no. 1, pp. 115-123, 2017.

[16] B.K. Bhattacharya, H.K. Sarmah and K.K. Sarma, "Implementing chaotic and synchronization properties of logistic maps using artificial neural networks for code generation," Advances in Intelligent Systems and Computing, vol. 638, pp. 409-418, 2018.

[17] U. Cavusoglu, A. Akgul, A. Zengin and I. Pehlivan, "The design and implementation of hybrid RSA algorithm using a novel chaos based RNG," Chaos, Solitons and Fractals, vol. 104, pp. 655-667, 2017.

[18] X. Wang, S. Wang, Y. Zhang and C. Luo, “A one-time pad color image cryptosystem based on SHA-3 and multiple chaotic systems," Optics and Lasers in Engineering, vol. 103, pp. 1-8, 2018.

[19] M. Jridi and A. Alfalou, "Real-time and encryption efficiency improvements of simultaneous fusion, compression and encryption method based on chaotic generators," Optics and Lasers in Engineering, vol. 102, pp. 59-69, 2018.

[20] K. Nosrati, C. Volos and A. Azemi, "Cubature Kalman filterbased chaotic synchronization and image encryption," Signal Processing: Image Communication, vol. 58, pp. 35-48, 2017.

[21] A.T. Azar and S. Vaidyanathan, Chaos Modeling and Control Systems Design, Springer, Berlin, Germany, 2015.

[22] S. Vaidyanathan and C. Volos, Advances and Applications in Chaotic Systems, Springer, Berlin, Germany, 2017.

[23] S. Vaidyanathan, C.K. Volos, K. Rajagopal, I.M. Kyprianidis and I.N. Stouboulos, “Adaptive backstepping controller design for the anti-synchronization of identical WINDMI chaotic systems with unknown parameters and its SPICE implementation," Journal of Engineering Science and Technology Review, vol. 8, no. 2, pp. 74-82, 2015.

[24] S. Vaidyanathan, "Adaptive synchronizatoin of novel 3-D chemical chaotic reactor systems," International Journal of
ChemTech Research, vol. 8, no. 7, pp. 159-171, 2015.

[25] S. Vaidyanathan, C.K. Volos and V.T. Pham, "Global chaos control of a novel nine-term chaotic system via sliding mode control," Studies in Computational Intelligence, vol. 576, pp. 571-590, 2015.

[26] U.E. Kocamaz, B. Cevher and Y. Uyaroglu, Control and synchronization of chaos with sliding mode control based on cubic reaching rule, Chaos, Solitons and Fractals, vol. 105, pp. 9298, 2017.

[27] R. Hajiloo, H. Salarieh and A. Alasty, Chaos control in delayed phase space constructed by the Takens embedding theory, Communications in Nonlinear Science and Numerical Simulation, vol. 54, pp. 453-465, 2018.

[28] Y. Wen, "Passive equivalence of chaos in Lorenz system," IEEE Transactions on Circuits and Systems, Part I: Fundamental Theory and Applications, vol. 46, pp. 876-878, 1999.

[29] Y.Z. Song, G.Z. Zhao and D.L. Qi, "Passive control of chaotic system with multiple strange attractors," Chinese Physics, vol. 15, pp. 2266-2270, 2006.

[30] F.Q. Wang and C.X. Liu, "Passive control of a 4-scroll chaotic system,” Chinese Physics, vol. 16, no. 4, pp. 946-950, 2007.

[31] F.Q. Wang and C.X. Liu, "Synchronization of hyperchaotic Lorenz system based on passive control," Chiense Physics, vol. 15, no. 9, pp. 1971-1975, 2006.

[32] F.Q. Wang and C.X. Liu, "Synchronization of unified chaotic system based on passive control," Physica D, vol. 225, pp. 55-60, 2007.

[33] X.J. Wu, J.S. Liu and G.R. Chen, “Chaos synchronization of Rikitake chaotic attractor usin the passive control technique," Nonlinear Dynamics, vol. 53, nos. 1-2, pp. 45-53, 2008.

[34] U.E. Kocamez and Y. Uyaroglu, "Synchronization of Vilnius chaotic oscillators with active and pasive control," Journal of Circuits, Systems and Computers, vol. 23, no. 7, Article ID 1450103, 2014.

[35] V.T. Pham, S. Jafari, C. Volos, A. Giakoumis, S. Vaidyanathan and T. Kapitaniak, "A chaotic system with equilibria located on the rounded square loop and its circuit implementation," IEEE Transactions on Circuits and Systems II: Express Briefs, vol. 63, no. 9, pp. 878-882, 2016.

[36] C. Volos, J.O. Maaita, S. Vaidyanathan, V.T. Pham, I. Stouboulos and I. Kyprianidis, "A novel four-dimensional hyperchaotic four-wing system with a saddle-focus equilibrium," IEEE Transactions on Circuits and Systems II: Express Briefs, vol. 64, no.3, pp. 339-343, 2017.

[37] S. Vaidyanathan, A. Sambas, M. Mamat and M. Sanjaya WS, "Analysis, synchronisation and circuit implementation of a novel jerk chaotic system and its application for voice encryption," International Journal of Modelling, Identification and Control, vol. 28, no. 2, pp. 153-166, 2017.

[38] X. Wang, S. Vaidyanathan, C. Volos, V.T. Pham and T. Kapitaniak, "Dynamics, circuit realization, control and synchronization of a hyperchaotic hyperjerk system with coexisting attractors," Nonlinear Dynamics, vol. 89, no. 3, pp. 1673-1687, 2017.

[39] Q. Gao and J. Ma, "Chaos and Hopf bifurcation of a finance system," Nonlinear Dynamics, vol. 58, nos. 1-2, pp. 209-216, 2009.

[40] S. Vaidyanathan, Ch. K. Volos, O. I. Tacha, I. M. Kyprianidis, I. N. Stouboulos and V.-T. Pham, "Analysis, control and circuit simulation of a novel 3-D finance chaotic system," Studies in 
Computational Intelligence, vol. 636, pp. 495-512, 2016.

[41] J.G. Jian, X.L. Deng and J.F. Wang, "Globally exponentially attractive set and synchronization of a class of chaotic finance system," 6th International Symposium on Neural Networks, Wuhan, China, Advances in Neural Networks, Lecture Notes in Computer Science, vol. 5551, pp. 253-261, 2009.

[42] G.L. Cai and M.Z. Yang, "Globally exponentially attractive set and synchronization of a novel three-dimensional chaotic finance system," Third International Conference on Information and Computing, ICIC, Wuxi, Jiangsu, China, vol. 2, pp. 70-73, 2010.

[43] X. S. Zhao, Z.B. Li, and S.A. Li. "Synchronization of a chaotic finance system," Applied Mathematics and Computation, vol. 217, no. 13, pp. 6031-6039, 2011.

[44] J. Li and C.R. Xie, "Synchronization of the modified financial chaotic system via a single controller," International Conference on Frontiers of Manufacturing Science and Measuring Technology, ICFMM2011, Chongqing, China, Advanced Materials Research, vol. 230, no. 1, pp. 1045-1048, 2011.

[45] U. E. Kocamaz, A. Goksu, H. Taskin and Y. Uyaroglu, "Synchronization of chaos in nonlinear finance system by means of sliding mode and passive control methods: A comparative study," Information Technology and Control, vol. 44, no. 2, pp. 172-181, 2015.

[46] J. M. Zheng, X. S. Li, and Y. Qiu, "A simple hybrid synchronization for a class of chaotic financial systems," Discrete Dynamics in Nature and Society, vol. 2017, Article ID: 9129605 , 2017.

[47] H. Bao, N. Wang, B. Bao, M. Chen, P. Jin and G. Wang, "Initial condition-dependent dynamics and transient period in memristor-based hypogenetic jerk system with four line equilibria," Communications in Nonlinear Science and Numerical Simulation, vol. 57, pp. 264-275, 2018.
[48] G. Zhang, J. Ma, A. Alsaedi, B. Ahmad and F. Alzahrani, "Dynamical behavior and application in Josephson Junction coupled by memristor," Applied Mathematics and Computation, vol. 321, pp. 290-299, 2018.

[49] S. Vaidyanathan, A. Sambas and M. Mamat, "Analysis, synchronisation and circuit implementation of a novel jerk chaotic system and its application for voice encryption," International Journal of Modelling, Identification and Control, vol. 28, pp. 153-166, 2017.

[50] A. Sambas, S. Vaidyanathan, M. Mamat, W. S. M. Sanjaya and R. P. Prastio, "Design, analysis of the Genesio-Tesi chaotic system and its electronic experimental implementation". International Journal of Control Theory and Applications, vol. 9, pp. 141-149, 2016.

[51] A. Sambas, M. Mamat, W. S. M. Sanjaya, Z. Salleh and F. S. Mohamad, "Secure communications based on the synchronization of the new Lorenz-like attractor circuit". Advanced Studies in Theoretical Physics, vol. 9, pp. 379-394, 2015.

[52] A. Sambas, Mujiarto, M. Mamat and W. S. M. Sanjaya, "Numerical simulation and circuit implementation for a sprott chaotic system with one hyperbolic sinusoidal nonlinearity". Far East Journal of Mathematical Sciences, vol. 102, pp. 1165-1177, 2017.

[53] S. Vaidyanathan, A. Sambas, M. Mamat and M. Sanjaya WS, "A new three-dimensional chaotic system with a hidden attractor, circuit design and application in wireless mobile robot", Archives of Control Sciences, vol. 27, no. 4, pp. 541-554, 2017.

[54] C.I. Byrnes, A. Isidori and J.C. Willems, "Passivity, feedback equivalence and the global stabilization of minimum phase nonlinear systems," IEEE Transactions on Automatic Control, vol. 36, pp. 1228-1240, 1991.

[55] H.K. Khalil, Nonlinear Systems, Pearson Education, Indiana, USA, 2001. 